\title{
Pentagonal shaped microstrip patch antenna in wireless capsule endoscopy system
}

\author{
Bondili Kohitha Bai ${ }^{1}$ and Anita Thakur ${ }^{2}$ \\ Department of electronics and communication Engineering, ASET, Amity \\ University, Noida. \\ kohitha@gmail. $\mathrm{com}^{1}$ and anni.thakur@gmail. $\mathrm{com}^{2}$
}

\begin{abstract}
Wireless capsule endoscopy is a best option for exploring inaccessible areas of small intestine for inspection of gastrointestinal tract. This technique brings less pain compare to conventional endoscopy technique. The wireless endoscopy system comprises of three main modules: an ingestible capsule that is swallowed by the patient, an external control unit and display device for image display. In this paper we proposed pentagonal shape microstrip patch antenna for wireless capsule endoscopy system. Inhibiting characteristics of a single microstrip patch like low gain, light weight, thin thickness and smaller bandwidth, make it more popular. This kind of antenna is aggressive miniaturized to meet the requirements of the wireless capsule endoscope. The simulation results show that the designed Circular Polarization $(C P)$ pentagonal shaped microstrip patch antenna gives axial ratio of 0.6023 at $2.38 \mathrm{GHz}$ and $\mathrm{CP}$ axial ratio bandwidth of $36 \mathrm{MHz}$ with $1.5 \%$. The antenna designed for wireless capsule endoscopy is a proposed one, which may work effectively when compared to other antennas in the capsule.
\end{abstract}

\section{Keywords}

Wireless capsule endoscopy, microstrip patch antenna, pentagonal and circular polarization $(C P)$.

\section{INTRODUCTION}

Endoscopy instrument is a prevalent one to visually examine the digestive tract nowadays. This is different from the conventional endoscopy technique, which brings great pain to the patients. The wireless endoscopy system lesser the pain and reduce the risk of operating method. Wireless capsule endoscopy system has become an accepted routine for inspection of gastrointestinal disorders such as cancers and polyps [1-2]. It can also examine the disease of the small intestine, but also be used for tissue samples and biopsy or treatment [3]. This system provides the opportunity to explore hidden areas of the small intestine that are not accessible by traditional endoscopy [4].This procedure do not disturb normal activities of the patient and also patient can perform their usual activities while being screened.

In Wireless endoscopy system, an ingestible capsule that is swallowed by the patient consists of a camera, control unit and a microstrip patch antenna. This microstrip patch antenna is low profile antennas, comfortable to planar and non-planar surfaces, simple and inexpensive to fabricate using modern printed technology. It is also relatively inexpensive to manufacture and design. For good antenna performance, a thick dielectric substrate having a low dielectric constant is

Natarajan Meghanathan, et al. (Eds): ITCS, SIP, JSE-2012, CS \& IT 04, pp. 47-54, 2012. 
desirable since this provides better efficiency, larger bandwidth and better radiation. However, such a configuration leads to a larger antenna size. In order to design a compact microstrip patch antenna, higher dielectric constants must be used which are less efficient and result in narrower bandwidth. Hence a compromise must be reached between antenna dimensions and antenna performance.

The frequency selection for wireless capsule endoscopy is difficult. The electrical property of human tissue affects the frequency. The magnetic permeability of human tissue and vacuum are same. But the permittivity and conductivity of human tissue depends on the frequency. Because of the high water content of human tissue, the absorption of electromagnetic power increases with frequency.

The design and simulation of circularly polarized microstrip patch antenna, which can be easily implemented by properly cutting a small sized section from a side of the equilateral-pentagon patch [5]. The pentagonal-shaped microstrip patch antenna can be designed with only one probe feed to get circular polarization and it can be used for many wireless applications at different frequencies [6]. This explains the antenna which is designed for endoscopy application at frequency $2.4 \mathrm{GHz}$.

This article explains firstly the wireless endoscopy system overview in section 2, Antenna analysis and design in section 3 and finally, it simulates the Designed antenna which gives $6.7 \mathrm{dBi}$ gain at $2.38 \mathrm{GHz}$ with good circular polarization in section 4 .

\section{WIRELESS ENDOSCOPY SYSTEM OVERVIEW}

Figure 1 with blocks illustrates the operation of the wireless endoscopy system. This system comprises of three main modules; an ingestible capsule, handheld data storage device and a computer for display and data storage or processing. The main blocks are capsule that is swallowed by the patient and an external control unit. The capsule passes through the digestive track in which there is a camera, control unit and antenna. The capsule collects images of the gastrointestinal tract. The external control unit with an antenna in it is collecting data from the capsule and controlling the operation of the capsule. The external unit is a wearable device that can be carried by the patient. This unit can be connected to a computer for enhanced image display and further analysis. A conceptual way of the wireless capsule is depicted in the system with five main modules: image sensor and lighting, control unit, wireless communication unit, power source and mechanical actuator [7].
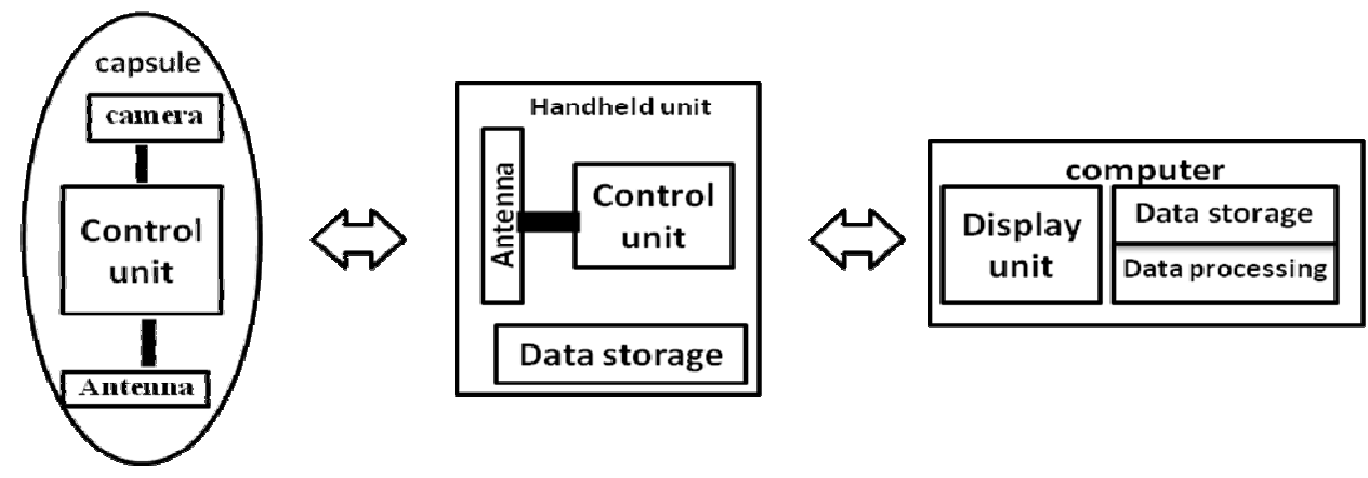

Figure 1. Block diagram of Wireless endoscopy system. 
The influence between human tissue and antenna is an important factor of propagating electromagnetic wave. During designing the antenna, the tissue must be considered. The tissue around the antenna could change its characteristics, such as the radiation efficiency, resonant frequency, input impedance and radiation mode. Characteristic parameters change along with different distance, different frequency, and other factors. Therefore, it is very important that the antenna is quantitatively analyzed by electromagnetic measurement to simulate the situation [7].

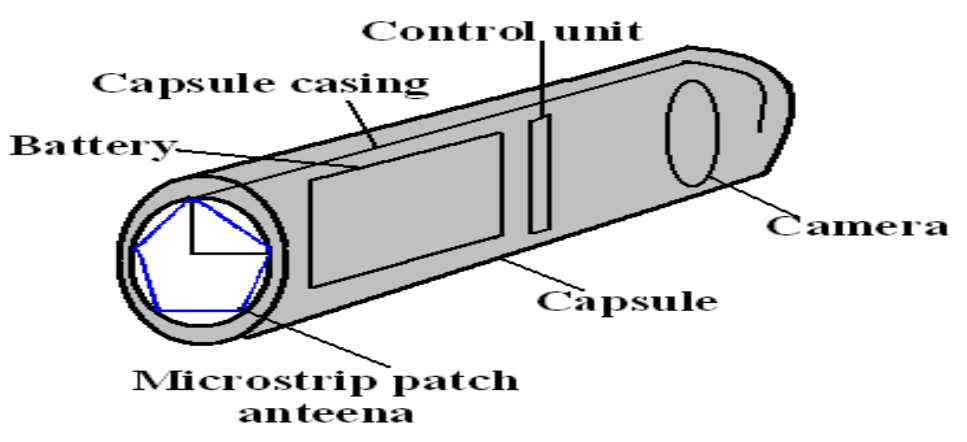

Figure 2. Diagram of endoscopy capsule system.

In endoscopy capsule system the main components as we pointed before, are designed as :camera at one end of capsule, on the other end microstrip patch antenna, in between antenna and camera they are control unit and battery as shown in figure 2. A capsule casing is a shell type casing for all components in a capsule.

\section{ANTENNA ANALYSIS}

Microstrip antennas are commonly used at frequencies from 1 to $100 \mathrm{GHz}$ and at frequencies below ultra high frequency, UHF microstrip patch become exceptionally large. The radiating patch of antenna can be designed in various shapes according to the desired characteristics like $\mathrm{H}$ shape micro strip antenna [8] and spring shape micro strip antenna [6]. In order to simplify analysis and performance prediction, the patch is generally square, rectangular, circular, triangular and elliptical or some other common shape.

Pentagonal microstrip patch gives better performance than the rectangular patch antenna. It also supports both linear and circular polarization. The pentagonal patch antenna gives circular polarization with only one feed where as rectangular patch antenna requires multiple feeds to get circular polarization [9]. The pentagonal patch antenna can also use multiple feeds and this type of antenna with multiple feeds can also give multiband operations [10].

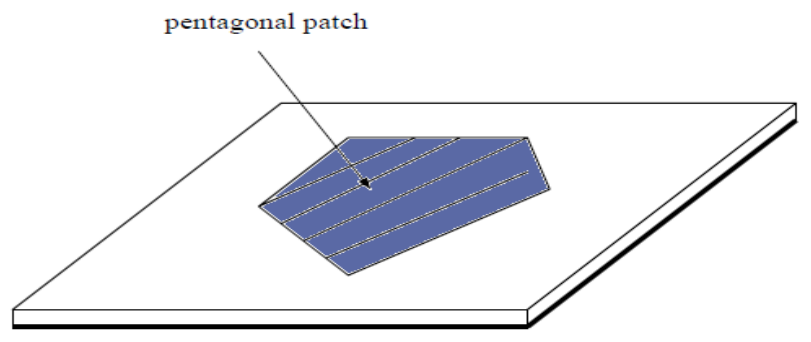

Figure 3. Geometry of a pentagonal patch. 
Circular polarization is theoretically possible from a microstrip antenna excited by a single feed if two spatially orthogonal modes are excited in phase quadrature. This can be achieved in a pentagonal patch as shown in Figure 3.

\subsection{Design of pentagon antenna}

The circularly polarized antenna which can be easily implement by properly slice a section $(\Delta \mathrm{L})$ from a side of the equilateral-pentagon patch in which the fundamental resonant mode of the equilateral - pentagon microstrip antenna and it is split into two near-degenerate orthogonal modes with equal amplitudes and a 90' phase difference [11]. The designed antenna which is operating at $2.4 \mathrm{GHz}$ with circular polarization has many advantages compared to the other microstrip patch antennas. This can be shown in figure 4 .

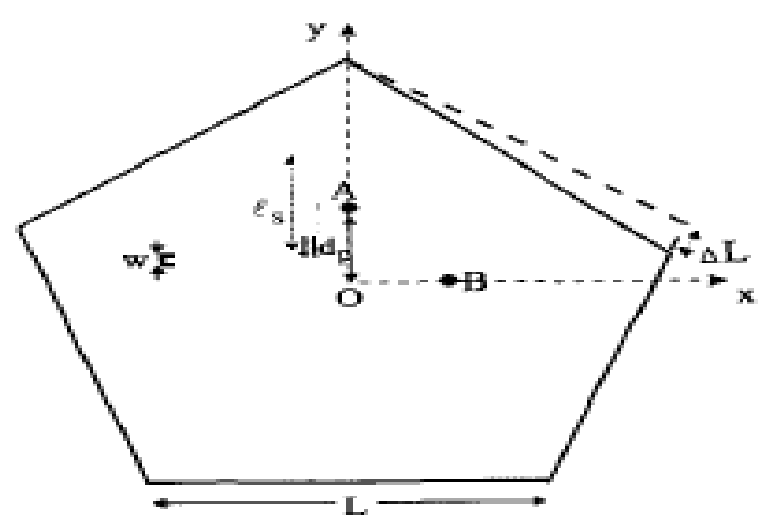

Figure 4. Equilqteral pentagon with $\Delta \mathbf{L}$.

\subsection{Design parameters}

The designed pentagonal microstrip patch antenna is a wideband small antenna with a thickness (h) of $3.2 \mathrm{~mm}$ and loss tangent of 0.0018 . The Substrate i.e.; considered for microstrip antenna is RT Duroid with dielectric constant $\varepsilon r=2.33$. This pentagonal patch design with feed point at $(8.8,-9.5)$ as in figure 5 , gives $6.7 \mathrm{dBi}$ gain at $2.38 \mathrm{GHz}$ with good circular polarization.

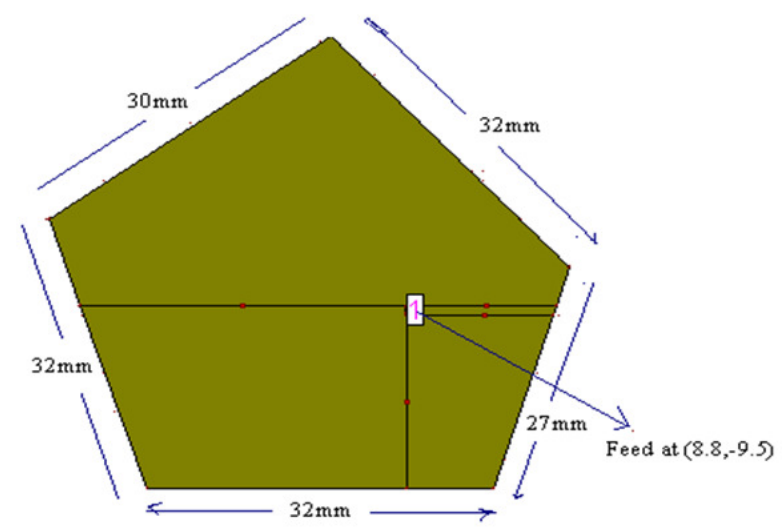

Figure 5. Geometry of the pentagonal patch. 
On the basis of analyzing the antenna and the miniaturization techniques, this paper adopts the resonant frequency of $2.4 \mathrm{GHz}$, feeding point and high-permittivity substrate to design the pentagonal patch antenna for wireless capsule endoscope as in figure 6.
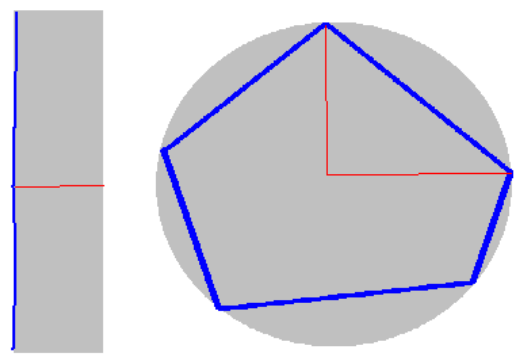

Figure 6. side and front view of pentagonal patch in capsule.

\section{SIMULATION RESULTS OF ANTENNA}

The following results are obtained from the ie $3 \mathrm{~d}$ simulation tool, these results are incorporating the antenna parameters as the designed pentagon shaped microstrip patch antenna gives reflection coefficient as shown in the figure 7. i.e., $\mathrm{S} 11(\mathrm{~dB})-31 \mathrm{~dB}$ which is minimum at resonant frequency of $2.38 \mathrm{GH}$. The radiation pattern is depicted from the figure 8 . The designed antenna is capable of generating circular polarization $(\mathrm{CP})$ with axial ratio of 0.6023 and $\mathrm{CP}$ axial ratio bandwidth of $1.5 \%$ as in figure 9 . And the antenna designed gives $\mathrm{CP}$ axial ratio-3 $\mathrm{dB}$ bandwidth of $36 \mathrm{MHz}$. Designed antenna gives $6.7 \mathrm{dBi}$ gain at $2.38 \mathrm{GHz}$ with good circular polarization which is pointed in figure 10.

\subsection{Reflection coefficient $(\mathrm{S} 11$ in $\mathrm{dB})$}

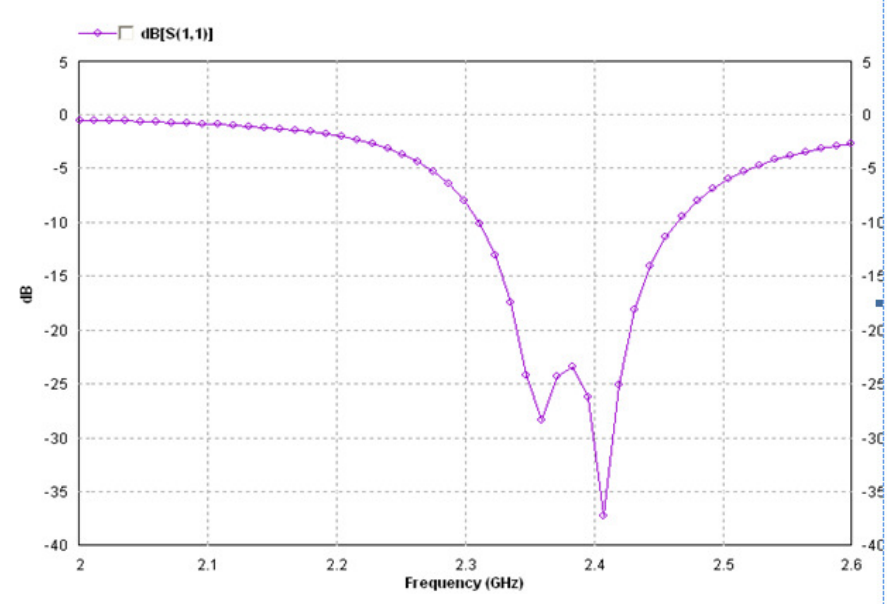

Figure 7. Reflection coefficient. 


\subsection{Radiation pattern}

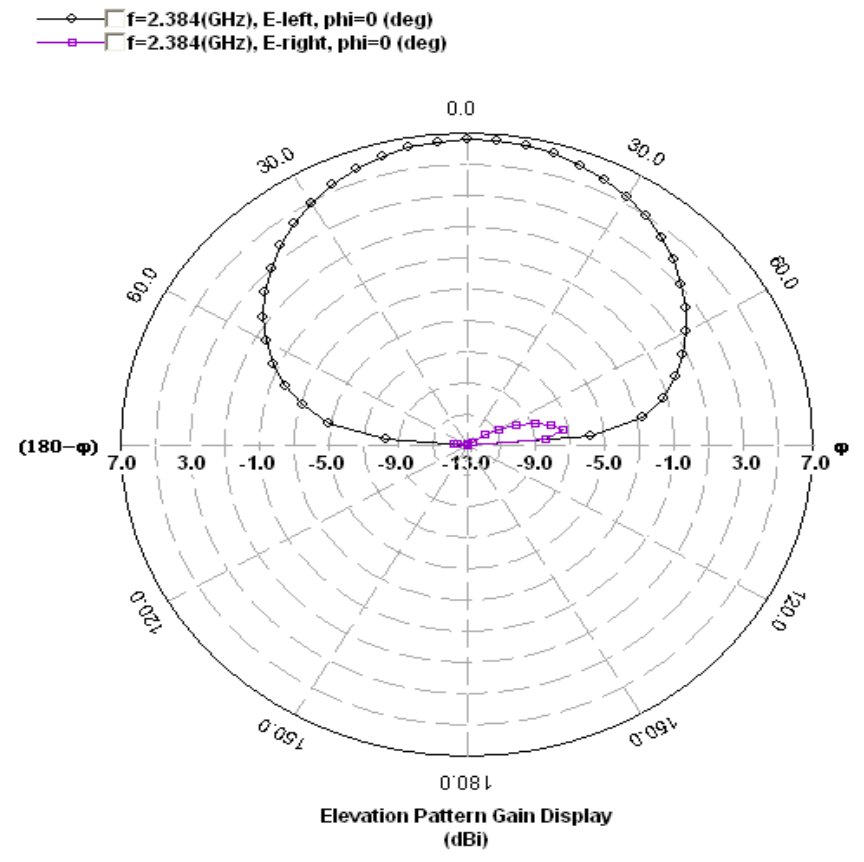

Figure 8. Radiation pattern.

\subsection{Axial ratio}

Axial-Ratio Vs. Frequency

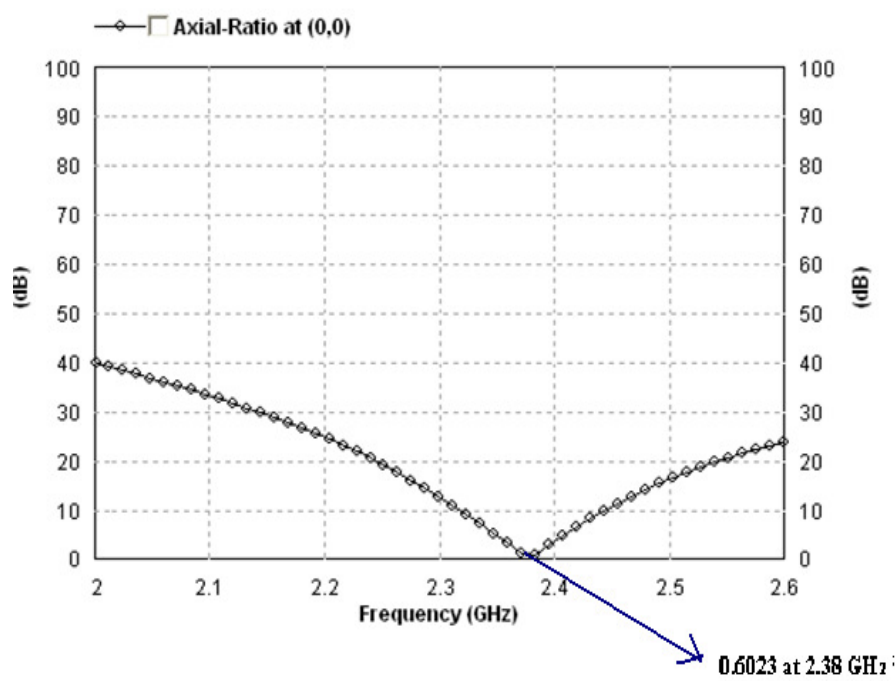

Figure 9. Axial ratio vs frequency. 


\subsection{Gain}

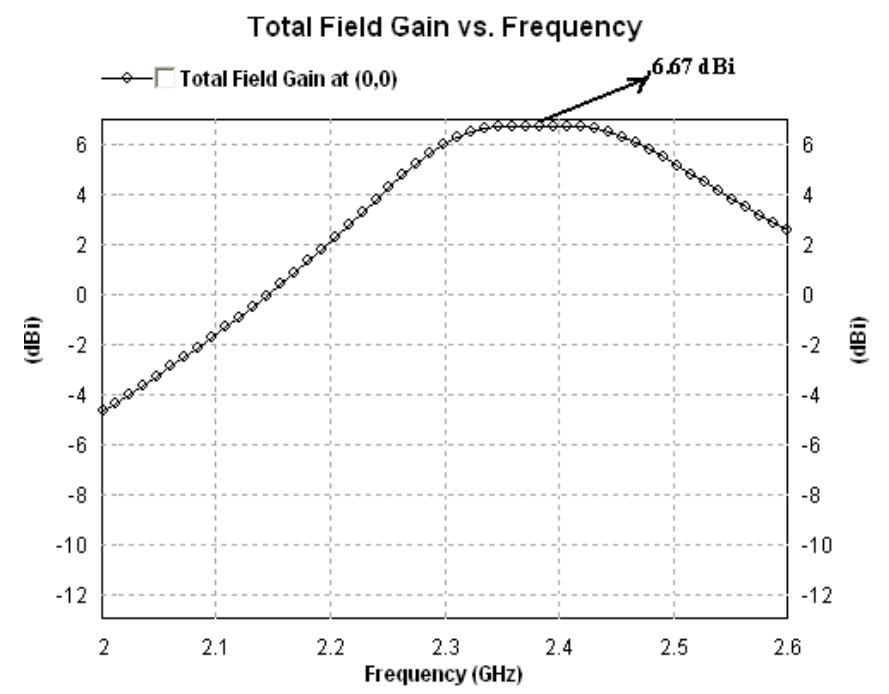

Figure 10. Gain vs. Frequency.

\section{CONCLUSION}

Ingestible wireless endoscopy capsule with pentagonal shape antenna design was proposed. The antenna has the following attractive features like miniaturized design for the capsule, good matching at the frequency of interest, wide band characteristics and easy human-body tuning. RFID bio-capsule tag was proposed for medical monitoring purposes. The antenna has the Omnidirectional radiation pattern. The proposed antenna gives an improved $\mathrm{CP}$ axial ratio bandwidth and minimum reflection coefficient. Designed antenna gives $6.7 \mathrm{dBi}$ gain at $2.38 \mathrm{GHz}$ with good circular polarization. Therefore, this antenna is suitable for wideband capsule endoscope systems.

\section{References}

[1] Moglia, A. Menciassi, M. O. Schurr, and P. Dario., "Wireless capsule endoscopy: from diagnostic devices to multipurpose robotic systems," Biomedical Microdevices, vol. 9, pp. 235-243, 2007.

[2] T. Nakamura and A. Terano, "Capsule endoscopy: past, present, and future," Journal of Gastroenterology, vol. 43, pp. 93-99, 2008.

[3] Sang Jun Moon, Yo Han Choi, and Seung S. Lee. "A Micromachined Biopsy Tool for a Capsule Type Endoscope[C]", 13th International Conference on Solid-State Sensors and Actuators and Microsystems, Seoul, South Korea, Jun 5-9 2005, pp1371-1374.

[4] D. S. Mishkin, et al., "ASGE Technology Status Evaluation Report on wireless capsule endoscopy," Gastrointestinal Endoscopy, vol. 63, pp.539-545, 2006.

[5] Wen-Shyang Chen and Horng-Dean Chen- "Compact circularly polarized pentagonal shaped microstrip antenna with bent losses" Antenna and propagation society international symposium, 2001 IEEE, Page(s):424-426, vol.3. 
[6] Noro, T.and Kazama, Y."A novel wideband circular polarization microstrip antenna - combination of different shaped antenna element". Antennas and Propagation Society International Symposium, 2005 IEEE Volume: 3A Publication Year: 2005, Page(s): 467 - 470 vol. 3A.

[7] Rasouli, M.Kencana, A.P. Van An Huynh Kiat, E. Lai, J.C.Y. Phee, L.S.J.,"Wireless Capsule Endoscopes for Enhanced Diagnostic Inspection of Gastrointestinal Tract" Conference on Robotics Automation and Mechatronics (RAM), 2010 IEEE Publication Year: 2010 , Page(s): 68 - 71.

[8] Performance of H-shaped microstrip antenna using IE3D, Kant Singh.R,Dhubkarya.P, D.C. Emerging Trends in Electronic and Photonic Devices \& Systems, 2009. International Conference on. 2009, on page(s): $364-366$.

[9] A novel wide beam circular polarization antenna - microstrip-dielectric antenna, He Haidan, Proceedings in Microwave and Millimeter Wave Technology, 2002. On page(s): 381 - 384 .

[10] Sang Heun Lee and Young Joong Yoon, "Fat Arm Spiral Antenna for Wideband Capsule Endoscope Systems" Conference on Bioinformatics and Biomedical Engineering (iCBBE), 2010 4th International Publication Year: 2010 , Page(s): 1 - 4.

[11] Natarajan and D. Chatterjee-"Effects of Ground Plane Shape on Performance of Probe-Fed, Circularly Polarized, Pentagonal Patch Antenna" Antennas and propagation Society International Symposium, 2003. IEEE, Page(s): 720 - 723. 